\title{
Effective simulation of quantum entanglement using classical fields modulated with pseudorandom phase sequences
}

\author{
Jian Fu*, and Xingkun Wu \\ Department of Optical Engineering, State Key Lab of Modern Optical Instrumentation, Zhejiang University, \\ Hangzhou 310027, China \\ *Corresponding author's e-mail address: jianfu@zju.edu.cn
}

Published online: 24 March 2015 (version 1)

Cite as: Fu J and Wu X, ScienceOpen Research 2015 (DOI: 10.14293/S2199-1006.1.SOR-PHYS.ANVYQZ.v1)

Reviewing status: Please note that this article is under continuous review. For the current reviewing status and the latest referee's comments please click here or scan the QR code at the end of this article.

\author{
Primary discipline: Quantum physics \& Field theory \\ Secondary discipline Theoretical physics \\ Keywords: Quantum Entanglement, Pseudorandom Phase Sequences, Classical Field \\ PACS: numbers 03.67.Lx, 03.65.Bz, 42.50.2p, 42.79.Ta
}

\begin{abstract}
An effective simulation of quantum entanglement is presented using classical fields modulated with $n$ pseudorandom phase sequences that constitute a $n 2^{n}$-dimensional Hilbert space with a tensor product structure. Applications to classical fields are examplied by effective simulation of both Bell and GHZ states, and a correlation analysis was performed to characterize the simulation. Results that strictly comply with criteria of quantum entanglement were obtained and the approach was also shown to be applicable to a system consisting of $n$ quantum particles.
\end{abstract}

Quantum entanglement, one of the most fascinating and important features in quantum theory, is widely appreciated as an essential ingredient in quantum computations [1-5]. Simulations of quantum entanglement through optical approaches were investigated both theoretically and experimentally [6-18]. A quantum bit can be represented by a distinct path or space mode of a classical field in an interferometric setup as classical optics analogies [9-18]. However, a $n$-qubit system with $2^{n}$ basis states must be represented by $2^{n}$ distinct paths or modes of a classical field. These simulations are usually not effective due to an exponential increase in required physical resources correlated with the addition of quantum bits [9-19]. The drawback can be traced to a lack of a rigorous tensor product structure of the system $[3,19,20]$.

In this letter, we present an effective simulation of quantum entanglement of $n$ quantum bits by using an analogy of classical fields modulated with pseudorandom phase sequences (PPSs). Based on the properties of PPSs, we proved that the $n$ fields modulated with $n$ different PPSs constitute a $n 2^{n}$-dimensional Hilbert space with a tensor product structure, which differs significantly from those in classical simulations that were executed lacking a tensor product structure [9-18]. By using an optical interferometric setup, PPSs yield not only random measurement results, but also an ensemble model to define the ensemble average and correlation functions [21]. The PPSs, derived from orthogonal pseudorandom sequences, are widely applied to code division multiple access (CDMA) communication technology as a way to distinguish different users [22-24]. A set of pseudorandom sequences is generated by using a shift register guided by a Galois field $G F(p)$ that satisfies orthogonal, closure, and balance properties [23]. In this letter, we utilize a m-sequence of period $N-1\left(N=p^{S}\right)$ generated by a primitive polynomial of degree $s$ over $G F(p)$ and apply it to 4-ary phase shift modulation, a well-known modulation format in wireless and optical communications [22, 24]. Next we generate a PPS set $\Xi=\left\{\lambda^{(1)}, \lambda^{(2)}, \ldots \lambda^{(N)}\right\}$ over $G F(4)$, where each $\lambda^{(i)}$ is a phase sequence with $N$ phase units and time slots: $\lambda^{(i)}=\left[\lambda_{1}^{(i)}, \lambda_{2}^{(i)}, \cdots \lambda_{N}^{(i)}\right]$, while $\lambda^{(1)}$ is an all-0 sequence and other sequences can be generated by using following method $[23,25]$ : (1) given a primitive polynomial of degree $s$ over $G F$ (4), a base sequence of a length $4^{s}-1$ is generated by the linear feedback shift register; (2) other sequences are obtained by cyclic shifting of the base sequence; (3) by adding zeroes to the sequences, the occurrence of any element equals to $4^{s}-1$; (4) mapping the elements of the sequences to $[0,2 \pi]$ : 0 mapping 0,1 mapping $\pi / 2,2$ mapping $\pi$, and 3 mapping $3 \pi / 2$.

We first consider two orthogonal modes (polarization or transverse modes), $|0\rangle$ and $|1\rangle$, of a classical field. A simulation state can be expressed as a mode superposition: $|\psi\rangle=\alpha|0\rangle+\beta|1\rangle$, where $|\alpha|^{2}+|\beta|^{2}=1,(\alpha, \beta \in \mathbb{C})$. All of the mode superposition states span a Hilbert space, where we will explore properties associated with this special classical field. By introducing a map $f: \lambda \rightarrow e^{i \lambda}$ on the set of $\Xi$, we obtain a 
phase sequence set $\Omega=\left\{\varphi^{(j)} \mid \varphi^{(j)}=e^{i \lambda^{(j)}}, j=1 \ldots N\right\}$, and with which were written a superposed state corresponding to $n$th sequence:

$$
\left|\psi_{n}\right\rangle \equiv e^{i \lambda^{(n)}}\left(\alpha_{n}|0\rangle+\beta_{n}|1\rangle\right)
$$

According to the properties of m-sequence, the set $\Omega$ has following properties: (a) closure: the product of any two sequences equals one of sequence in the set; (b) balance: except $\varphi^{(1)}$, any sequence of set $\Omega$ satisfy $\sum_{k=1}^{N} e^{i \theta} \varphi_{k}^{(j)}=\sum_{k=1}^{N} e^{i\left(\theta+\lambda_{k}^{(j)}\right)}=0, \forall \theta \in \mathbb{R}$; (c) orthogonality: any two of the sequences satisfy normalized correlation: $E\left(\varphi^{(i)}, \varphi^{(j)}\right)=\frac{1}{N} \sum_{k=1}^{N} \varphi_{k}^{(i)} \varphi_{k}^{(j) *}$, which equals 1 when $i=j$, and 0 otherwise. In fact, the map $f$ corresponds to phase modulations of PPSs of $\Xi$ onto the classical field.

A PPS map $f$ constitutes a phase ensemble, wherein each phase unit represents a single simulation, and measurement of a physical quantity is a result of ensemble average. Similar to that in quantum mechanics, ensemble average and correlation measurement can be defined [22-24]. In the quadrature demodulation, each code obtained in a sequence unit of a PPS can be considered as a single measurement. The sequence number of the PPS's unit can be used to label the ensemble. Different from the ergodicity hypothesis of quantum mechanics, the ergodicity of PPS is determined and much more efficient.

Given the properties of the PPSs and the Hilbert space, the inner product of any two states and their orthogonal property can be obtained by:

$$
\left\langle\psi_{i} \mid \psi_{j}\right\rangle=\frac{1}{N} \sum_{k=1}^{N} e^{i\left(\lambda_{k}^{(j)}-\lambda_{k}^{(i)}\right)}\left(\alpha_{i}^{*} \alpha_{j}+\beta_{i}^{*} \beta_{j}\right)=\left\{\begin{array}{l}
1, i=j \\
0, i \neq j
\end{array}\right.
$$

where $\lambda_{k}^{(i)}, \lambda_{k}^{(j)}$ are the $k$ th units of $\lambda^{(i)}$ and $\lambda^{(j)}$, respectively. Based on above properties, the classical fields modulated with different PPSs are independent and distinguishable. Figure 1 shows construction pathway of simulation states, generated by unitary transformed from initial states-the mode superposition of classical fields with PPS $\lambda^{(j)}$. Furthermore, a general form of a simulation state can be constructed from $\left|\psi_{n}\right\rangle$ :

$$
\left|\psi_{n}\right\rangle=\sum_{i=1}^{N} \alpha_{n}^{(i)} e^{i \lambda^{(i)}}|0\rangle+\sum_{j=1}^{N} \beta_{n}^{(j)} e^{i \lambda^{(j)}}|1\rangle
$$

Following the pathway in Figure 1, a simulation state $|\Psi\rangle$ is obtained, denoting with a direct product of $\left|\psi_{n}\right\rangle$ :

$$
|\Psi\rangle=\left|\psi_{1}\right\rangle \otimes\left|\psi_{2}\right\rangle \ldots \otimes\left|\psi_{N}\right\rangle
$$

Due to closure property of PPS, the phase sequence $e^{i \lambda^{(j)}}$ of each state $\left|i_{1} i_{2} \ldots i_{N}\right\rangle$ remains to belong to 0 and consists of the product of multiple sequences. Basis for Hilbert space of simulation is spanned by $\left\{e^{i \lambda^{(j)}}\left|i_{1} i_{2} \ldots i_{N}\right\rangle \mid j=1 \ldots N, i_{n}=0\right.$ or 1$\}$, with a total base state number of $N 2^{N}$. Generally a simulation state takes the form:

$$
|\Psi\rangle=\sum_{i_{1}=0}^{1} \ldots \sum_{i_{N}=0}^{1}\left[\sum_{j=1}^{N} C_{i_{1} \ldots i_{N}}^{(j)} e^{i \lambda^{(j)}}\left|i_{1} i_{2} \ldots i_{N}\right\rangle\right]
$$

where $C_{i_{1} \ldots i_{N}}^{(j)}$ denotes a total of $N 2^{N}$ coefficients. It is obvious that the Hilbert simulation space is greater than what is required for simulation of quantum state. To obtain a space the same size as that in quantum mechanics, either restrictions or a proper measurement need to apply [21].

PPS provides not only the tensor structure and space needed for quantum state simulation, it also yields the property that an entangled state cannot be expressed in terms of direct product of tensors by using PPS properties and phase ensemble average. In the following we use density matrix to illustrate this feature. We assume that a simple type of simulation state of $N$ fields can be expressed as:

$$
\begin{aligned}
|\Psi\rangle & =\left|\psi_{1}\right\rangle \otimes\left|\psi_{2}\right\rangle \ldots \otimes\left|\psi_{N}\right\rangle \\
& =e^{i \lambda^{\text {sum }}}\left(\sum_{i=1}^{N^{\prime}} C_{i}\left|x_{i}\right\rangle+\sum_{j=1}^{N^{\prime \prime}} C_{j} e^{i \lambda^{(j)}}\left|x_{j}\right\rangle\right)
\end{aligned}
$$

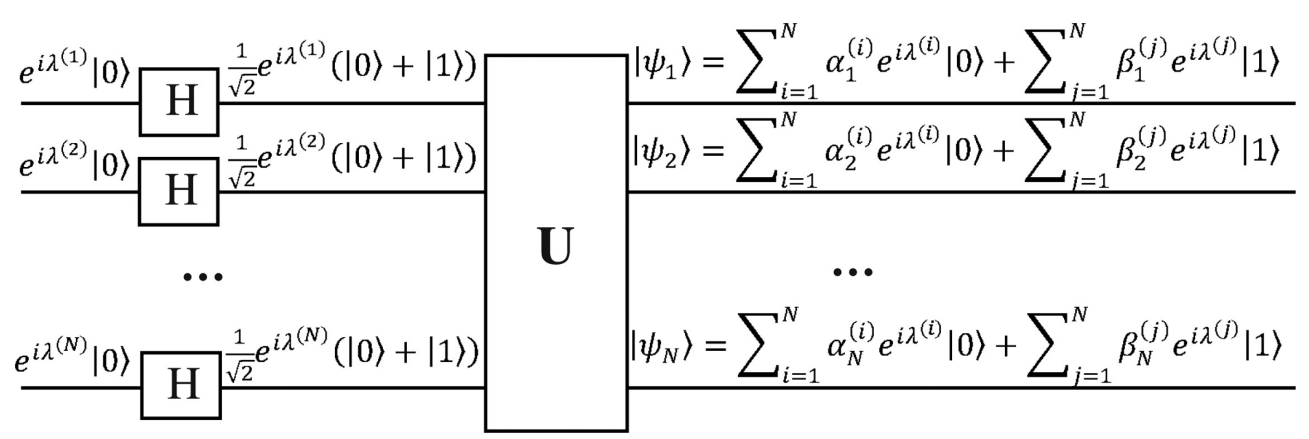

Figure 1. Construction pathway of simulation states is displayed, which are brought by unitary transformed from initial states-the mode superposition of classical fields with PPS $\lambda^{(i)}$. 
where $\quad N^{\prime}+N^{\prime \prime}=2^{N} \quad$ and $\quad N^{\prime \prime}<N, \quad \lambda^{\text {sum }}=\sum_{n=1}^{N} \lambda^{(n)}$, and $|x\rangle=\left|i_{1} i_{2} \ldots i_{N}\right\rangle$. A density matrix $\rho$ can be calculated by:

$$
\begin{aligned}
\rho \equiv|\Psi\rangle\langle\Psi|= & e^{i \lambda^{\text {sum }}}\left(\sum_{i=1}^{N^{\prime}} C_{i}\left|x_{i}\right\rangle+\sum_{j=1}^{N^{\prime \prime}} C_{j} e^{i \lambda^{(j)}}\left|x_{j}\right\rangle\right) \\
& \times e^{-i \lambda^{\text {sum }}}\left(\sum_{i=1}^{N^{\prime}} C_{i}^{*}\left\langle x_{i}\right|+\sum_{j=1}^{N^{\prime \prime}} C_{j}^{*} e^{-i \lambda^{(j)}}\left\langle x_{j}\right|\right)
\end{aligned}
$$

which is simplified into

$$
\begin{aligned}
\rho= & \sum_{n=1}^{2^{N}}\left|C_{n}\right|^{2}\left|x_{n}\right\rangle\left\langle x_{n}\right|+\sum_{i \neq i^{\prime}=1}^{N^{\prime}}\left(C_{i^{\prime}}^{*} C_{i}\left|x_{i}\right\rangle\left\langle x_{i^{\prime}}\left|+C_{i}^{*} C_{i^{\prime}}\right| x_{i^{\prime}}\right\rangle\left\langle x_{i}\right|\right) \\
& +\sum_{j \neq j^{\prime}=1}^{N^{\prime \prime}}\left(C_{j^{\prime}}^{*} C_{j} e^{i \lambda^{(l)}}\left|x_{j}\right\rangle\left\langle x_{j^{\prime}}\left|+C_{j}^{*} C_{j^{\prime}} e^{-i \lambda^{(l)}}\right| x_{j^{\prime}}\right\rangle\left\langle x_{j}\right|\right) \\
& +\sum_{i=1}^{N^{\prime}} \sum_{j=1}^{N^{\prime \prime}}\left(C_{i}^{*} C_{j} e^{i \lambda^{(j)}}\left|x_{j}\right\rangle\left\langle x_{i}\left|+C_{j}^{*} C_{i} e^{-i \lambda^{(j)}}\right| x_{i}\right\rangle\left\langle x_{j}\right|\right)
\end{aligned}
$$

where $\lambda^{(l)}=\lambda^{(j)}-\lambda^{\left(j^{\prime}\right)}$. By applying phase ensemble averaging [23], mean reduced density matrix is defined $\tilde{\rho} \equiv \frac{1}{N} \sum_{k=1}^{N} \rho$. Due to balance property of PPS $\sum_{k=1}^{N} e^{i \lambda_{k}^{(l)}}=0$, then we obtain:

$$
\tilde{\rho}=\sum_{n=1}^{2^{N}}\left|C_{n}\right|^{2}\left|x_{n}\right\rangle\left\langle x_{n}\right|+\sum_{i \neq i^{\prime}=1}^{N^{\prime}}\left(C_{i^{\prime}}^{*} C_{i}\left|x_{i}\right\rangle\left\langle x_{i^{\prime}}\left|+C_{i}^{*} C_{i^{\prime}}\right| x_{i^{\prime}}\right\rangle\left\langle x_{i}\right|\right)
$$

Equation (9) shows that all non-diagonal terms including $\left|x_{j}\right\rangle$ disappear and the reduced density matrix $\tilde{\rho}$ might not be expressed in terms of a direct product of the states $\left|x_{n}\right\rangle$, similar to the case of quantum entanglement states.

In addition to the fact that a quantum entanglement cannot be expressed in terms of direct tensor product, quantum entanglement also makes a correlation measurement different. The correlation analysis on the simulation states is necessary because the nonlocal correlation with Bell's inequality and equality criterion is the most fundamental property of quantum entanglement. In order to perform the correlation analysis, a correlation measurement $\widehat{P}$ on $|\psi\rangle$ is given:

$$
\begin{aligned}
\bar{P}(\theta) & =\langle\psi|\widehat{P}(\theta)| \psi\rangle=\left(\begin{array}{ll}
\alpha^{*} & \beta^{*}
\end{array}\right)\left(\begin{array}{cc}
0 & e^{i \theta} \\
e^{-i \theta} & 0
\end{array}\right)\left(\begin{array}{l}
\alpha \\
\beta
\end{array}\right) \\
& =\alpha^{*} \beta e^{i \theta}+\alpha \beta^{*} e^{-i \theta}
\end{aligned}
$$

For convenience, coefficients $\alpha, \beta$ are set to be $1 / \sqrt{2}$, yielding $P(\theta)=\cos (\theta)$. Furthermore, we generalize $\widehat{P}$ to the case of $N$ fields:

$$
\widehat{P}\left(\theta_{1}, \ldots \theta_{N}\right)=\widehat{P}\left(\theta_{1}\right) \otimes \widehat{P}\left(\theta_{2}\right) \otimes \ldots \widehat{P}\left(\theta_{N}\right)
$$

Then we obtain the correlation analysis of the simulation states using $\widehat{P}$ and the density matrix $\rho$ :

$$
\begin{aligned}
E\left(\theta_{1}, \ldots \theta_{N}\right)= & \frac{1}{N} \sum_{k=1}^{N} \operatorname{Tr}\left[\rho \widehat{P}\left(\theta_{1}, \ldots \theta_{N}\right)\right] \\
= & \operatorname{Tr}\left[\tilde{\rho} \widehat{P}\left(\theta_{1}, \ldots \theta_{N}\right)\right]=\sum_{n=1}^{2^{N}}\left|C_{n}\right|^{2}\left\langle x_{n}|\widehat{P}| x_{n}\right\rangle \\
& +\sum_{i \neq i^{\prime}=1}^{N^{\prime}}\left(C_{i^{\prime}}^{*} C_{i}\left\langle x_{i^{\prime}}|\widehat{P}| x_{i}\right\rangle+C_{i}^{*} C_{i^{\prime}}\left\langle x_{i}|\widehat{P}| x_{i^{\prime}}\right\rangle\right)
\end{aligned}
$$

Equation (12) shows that only non-diagonal terms $\sum_{i \neq i^{\prime}=1}^{N^{\prime}}\left(C_{i^{\prime}}^{*} C_{i}\left\langle x_{i^{\prime}}|\widehat{P}| x_{i}\right\rangle+C_{i}^{*} C_{i^{\prime}}\left\langle x_{i}|\widehat{P}| x_{i^{\prime}}\right\rangle\right)$ remain.

Key to an effective simulation of quantum entanglement is that the physical resources for the simulation do not increase exponentially with number of particle. In the following we discuss analysis of computation complexity. A simple unitary transformation, NOT gate, is used as an example to show computation complexity. Starting with a single field $\left|\psi_{n}\right\rangle=e^{i \lambda^{(n)}}\left(\alpha_{n}|0\rangle+\beta_{n}|1\rangle\right)$, applying a unitary transformation switching $\widehat{U}:|0\rangle \leftrightarrow|1\rangle$ to decomposes PPS into each phase unit: $\widehat{U}\left|\psi_{n}\right\rangle \rightarrow\left[e^{i \lambda_{k}^{(n)}} \widehat{U}\left(\alpha_{n}|0\rangle+\beta_{n}|1\rangle\right) \mid k=1 \ldots N\right]=$ $e^{i \lambda^{(n)}}\left(\alpha_{n}|1\rangle+\beta_{n}|0\rangle\right)$. For each phase unit, its computation is the same as that in quantum computation, therefore computation for $N$ phase units equals $N$ times of quantum computation of each phase unit. We can extend unitary transformations to simulation states with $N$ fields:

$$
\begin{aligned}
& \widehat{U}:|\Psi\rangle \rightarrow\left|\Psi^{\prime}\right\rangle \\
& |\Psi\rangle=\sum_{i_{1}=0}^{1} \ldots \sum_{i_{N}=0}^{1}\left[\sum_{j=1}^{N} C_{i_{1} \cdots i_{N}}^{(j)} e^{i \lambda^{(j)}}\left|i_{1} \ldots i_{k} \ldots i_{N}\right\rangle\right] \\
& \left|\Psi^{\prime}\right\rangle=\sum_{i_{1}=0}^{1} \ldots \sum_{i_{N}=0}^{1}\left[\sum_{j=1}^{N} C_{i_{1} \cdots i_{N}}^{(j)^{\prime}} e^{i \lambda^{(j)}}\left|i_{1} \ldots i_{k}^{\prime} \ldots i_{N}\right\rangle\right]
\end{aligned}
$$

and coefficients $C_{i_{1} \cdots i_{N}}^{(j)}$ and $C_{i_{1} \cdots i_{N}}^{(j)^{\prime}}$ are related by an unitary transformation:

$$
C_{i_{1} \cdots i_{k}^{\prime} \cdots i_{N}}^{(j)^{\prime}}=\sum_{i_{k}} U_{i_{k}^{\prime}}^{i_{k}} C_{i_{1} \cdots i_{k} \cdots i_{N}}^{(j)}
$$

Because a PPS contains $N$ phase units $e^{i \lambda_{k}^{(j)}}$ and $N$ time slots, therefore the required computation is $N$ times that of quantum computation, but $2^{N}$ times is unnecessary [3].

Two-particles Bell states: Consider the case that the modes $|1\rangle$ in the states $\left|\psi_{a}\right\rangle$ and $\left|\psi_{b}\right\rangle$ similar to Equation (1) are exchanged by a mode exchanger constituted by mode splitters and combiners $[21,26]$. The exchange yields the following states:

$$
\begin{aligned}
& \left|\psi_{a}^{\prime}\right\rangle=\frac{e^{i \lambda^{(a)}}}{\sqrt{2}}\left(|0\rangle+e^{i \gamma^{(a)}}|1\rangle\right) \\
& \left|\psi_{b}^{\prime}\right\rangle=\frac{e^{i \lambda^{(b)}}}{\sqrt{2}}\left(|0\rangle+e^{i \gamma^{(b)}}|1\rangle\right)
\end{aligned}
$$


where the relative phase sequences (RPSs) $\gamma^{(a)}=-\gamma^{(b)}=$ $\lambda^{(b)}-\lambda^{(a)}$, and $\gamma^{(a)}+\gamma^{(b)}=0$.

The simulation state $|\Psi\rangle$ is obtained:

$$
\begin{aligned}
|\Psi\rangle & =\left|\psi_{a}^{\prime}\right\rangle \otimes\left|\psi_{b}^{\prime}\right\rangle \\
& =\frac{e^{i\left(\lambda^{(a)}+\lambda^{(b)}\right)}}{2}\left[|0\rangle|0\rangle+|1\rangle|1\rangle+e^{i \gamma^{(a)}}|1\rangle|0\rangle+e^{i \gamma^{(b)}}|0\rangle|1\rangle\right]
\end{aligned}
$$

Apparently the reduced density matrix $\tilde{\rho}$ cannot be direct product decomposed due to only non-diagonal term $|00\rangle \times$ $\langle 11|+| 11\rangle\langle 00|$ remaining.

Then we obtain the results of the fields in the correlation measurement $\bar{P}\left(\theta_{a}, k\right)=\cos \left(\theta_{a}+\gamma_{k}^{(a)}\right) \quad$ and $\bar{P}\left(\theta_{b}, k\right)=$ $\cos \left(\theta_{b}+\gamma_{k}^{(b)}\right)$, where $\gamma_{k}^{(a)}, \gamma_{k}^{(b)}$ are the $k$ th units of the RPSs $\gamma^{(a)}$ and $\gamma^{(b)}$, respectively. Then the correlation function is:

$$
E\left(\theta_{a}, \theta_{b}\right)=\frac{1}{N C} \sum_{k=1}^{N} \bar{P}\left(\theta_{a}, k\right) \bar{P}\left(\theta_{b}, k\right)=\cos \left(\theta_{a}+\theta_{b}\right)
$$

where $C=1 / 2$ is the normalization coefficient. The states in Equation (15) are considered to be a classical field simulation of the Bell state $\left|\Psi^{+}\right\rangle$. By substituting the above correlation functions into Bell inequality (CHSH inequality) [27]:

$$
\begin{aligned}
|B| & =\left|E\left(\theta_{a}, \theta_{b}\right)-E\left(\theta_{a}, \theta_{b}^{\prime}\right)+E\left(\theta_{a}^{\prime}, \theta_{b}^{\prime}\right)+E\left(\theta_{a}^{\prime}, \theta_{b}\right)\right| \\
& =2 \sqrt{2}>2
\end{aligned}
$$

where $\theta_{a}, \theta_{a}^{\prime}, \theta_{b}$ and $\theta_{b}^{\prime}$ are $\pi / 4,-\pi / 4,0$ and $\pi / 2$, respectively, when Bell's inequality is maximally violated.

Bell state $\left|\Psi^{+}\right\rangle$differs from $\left|\Psi^{-}\right\rangle$by $\pi$ phase. Similarly, simulation of the Bell state $\left|\Psi^{-}\right\rangle$is expressed as $\left|\psi_{a}^{\prime}\right\rangle=$ $e^{i \lambda^{(a)}}\left(|0\rangle+e^{i \gamma^{(a)}}|1\rangle\right) / \sqrt{2},\left|\psi_{b}^{\prime}\right\rangle=e^{i \lambda^{(b)}}\left(|0\rangle+e^{i\left(\gamma^{(b)}+\pi\right)}|1\rangle\right) / \sqrt{2}$. By performing the transformation $\widehat{\sigma}_{x}:|0\rangle \leftrightarrow|1\rangle$ on $\left|\psi_{b}^{\prime}\right\rangle$ of the state $\left|\Psi^{ \pm}\right\rangle$, we obtain the simulation of the Bell state $\left|\Phi^{+}\right\rangle$expressed as $\left|\psi_{a}^{\prime}\right\rangle=e^{i \lambda^{(a)}}\left(|0\rangle+e^{i \gamma^{(a)}}|1\rangle\right) / \sqrt{2},\left|\psi_{b}^{\prime}\right\rangle=$ $e^{i \lambda^{(b)}}\left(|1\rangle+e^{i \gamma^{(b)}}|0\rangle\right) / \sqrt{2}$, and of $\left|\Phi^{-}\right\rangle$expressed as $\left|\psi_{a}^{\prime}\right\rangle=$ $e^{i \lambda^{(a)}}\left(|0\rangle+e^{i \gamma^{(a)}}|1\rangle\right) / \sqrt{2},\left|\psi_{b}^{\prime}\right\rangle=e^{i \lambda^{(b)}}\left(|1\rangle+e^{i\left(\gamma^{(b)}+\pi\right)}|0\rangle\right) / \sqrt{2}$. Then their correlation functions $E_{\Psi^{-}}\left(\theta_{a}, \theta_{b}\right)=-\cos \left(\theta_{a}+\theta_{b}\right)$, $E_{\Phi^{ \pm}}\left(\theta_{a}, \theta_{b}\right)= \pm \cos \left(\theta_{a}-\theta_{b}\right)$ are obtained. To substitute the correlation functions into Equation (18), we also obtain the maximal violation of Bell's inequality. The violation of Bell's criterion demonstrates the nonlocal correlation of the two classical fields in our simulation, which results from shared randomness of the PPSs.

GHZ states: The nonlocality of the multipartite entangled GHZ states can in principle be manifest in a new criterion and need not be statistical as the violation of Bell inequality [28]. Preparing three states $\left|\psi_{a}\right\rangle,\left|\psi_{b}\right\rangle$ and $\left|\psi_{c}\right\rangle$ similar to Equation (1), and by cyclically exchanging the modes $|1\rangle$ of the states, we obtain the states as following:

$$
\begin{aligned}
& \left|\psi_{a}^{\prime}\right\rangle=\frac{e^{i \lambda^{(a)}}}{\sqrt{2}}\left(|0\rangle+e^{i \gamma^{(a)}}|1\rangle\right) \\
& \left|\psi_{b}^{\prime}\right\rangle=\frac{e^{i \lambda^{(b)}}}{\sqrt{2}}\left(|0\rangle+e^{i \gamma^{(b)}}|1\rangle\right) \\
& \left|\psi_{c}^{\prime}\right\rangle=\frac{e^{i \lambda^{(c)}}}{\sqrt{2}}\left(|0\rangle+e^{i \gamma^{(c)}}|1\rangle\right)
\end{aligned}
$$

where the RPSs $\gamma^{(a)}=\lambda^{(b)}-\lambda^{(a)}, \gamma^{(b)}=\lambda^{(c)}-\lambda^{(b)}, \gamma^{(c)}=\lambda^{(a)}-$ $\lambda^{(c)}$ and $\gamma^{(a)}+\gamma^{(b)}+\gamma^{(c)}=0$. We obtain the measurement results $\quad \bar{P}\left(\theta_{a}, k\right)=\cos \left(\theta_{a}+\gamma_{k}^{(a)}\right), \bar{P}\left(\theta_{b}, k\right)=\cos \left(\theta_{b}+\gamma_{k}^{(b)}\right)$, $\bar{P}\left(\theta_{c}, k\right)=\cos \left(\theta_{c}+\gamma_{k}^{(c)}\right)$ for the states $\left|\psi_{a}^{\prime}\right\rangle,\left|\psi_{b}^{\prime}\right\rangle$ and $\left|\psi_{c}^{\prime}\right\rangle$ in the correlation measurement, respectively, and the correlation function:

$$
\begin{aligned}
E\left(\theta_{a}, \theta_{b}, \theta_{c}\right) & =\frac{1}{N C} \sum_{k=1}^{N} \bar{P}\left(\theta_{a}, k\right) \bar{P}\left(\theta_{b}, k\right) \bar{P}\left(\theta_{c}, k\right) \\
& =\cos \left(\theta_{a}+\theta_{b}+\theta_{c}\right)
\end{aligned}
$$

where $C=1 / 4$ is the normalized coefficient. If $\theta_{a}+\theta_{b}+\theta_{c}=$ $0, E\left(\theta_{a}, \theta_{b}, \theta_{c}\right)=1$. If $\theta_{a}+\theta_{b}+\theta_{c}=\pi, E\left(\theta_{a}, \theta_{b}, \theta_{c}\right)=-1$. By using GHZ state, the family of simple proofs of Bell's theorem without inequalities can be obtained [29], which is different from the criterion of $\mathrm{CHSH}$ inequality. The sign of the correlation function can be also treated as the criterion, such as the negative correlation for nonlocal and the positive correlation for local when $\theta_{a}=\pi / 3, \theta_{b}=\pi / 3, \theta_{c}=\pi / 3$. We obtain that the simulation state in Equation (19) shows the negative correlation. The results are similar to the quantum case of GHZ states.

Furthermore, the simulation of GHZ state could be generalized to the case of $N$ particles. By preparing $N$ states similar to Equation (1) and cyclically exchanging the modes $|1\rangle$ of the states, the RPSs satisfy $\gamma^{(1)}+\cdots+\gamma^{(N)}=0$. We obtain the correlation function:

$$
\begin{aligned}
E\left(\theta_{1}, \ldots \theta_{N}\right) & =\frac{1}{N C} \sum_{k=1}^{N} \bar{P}\left(\theta_{1}, k\right) \ldots \bar{P}\left(\theta_{N}, k\right) \\
& =\cos \left(\theta_{1}+\cdots+\theta_{N}\right)
\end{aligned}
$$

where $\bar{P}\left(\theta_{i}, k\right)=\cos \left(\theta_{i}+\gamma_{k}^{(i)}\right)$ is the result of the classical field with $i$ th RPSs at the $k$ th sequence units in the correlation measurement, and $C=1 / 2^{N-1}$ is the normalized coefficient. Using the same notion, we can obtain simulation results of other quantum entanglement states. It should be pointed out that the phase randomness provided by PPSs is different from the case of quantum mixed states. Quantum mixed states result from decoherence and all coherent superposition items disappear. In contract to the decoherence, some coherent superposition items remain in the simulation state due to the constraints of the RPSs, such as $\gamma^{(a)}+\gamma^{(b)}=0, \gamma^{(a)}+\gamma^{(b)}+$ $\gamma^{(c)}=0$ for the simulation of Bell states and GHZ state, respectively. These remaining items make it possible to simulate quantum entangled pure states. 
As shown in the above examples, we utilized the properties of PPSs to label classical fields that are even overlapped in the same space and time. In simulation of entangled states, the resources required are the PPSs instead of classical field modes. It means that the amount of PPSs grows linearly with the number of quantum particles. According to the m-sequence theory, the number of PPSs in the set equals to the length of sequences, which means that the time resource (the length of sequence) required also grows linearly with the number of the particles.

In conclusion, a novel simulation method for quantum entanglement is presented, with its mathematical expressions and physical meanings identical to those in quantum mechanics. In the framework of quantum mechanics, the overall phase of a wave function can be ignored, as it has no contribution to the probability distribution. However, quantum entanglement must be related to two or more spatially separable quantum particles. By introducing a phase factor to superposed states with PPS properties, we conclude that quantum entanglement can be efficiently simulated by using a classical field modulated with PPSs. The research on this simulation not only provides useful insights into fundamental features of quantum entanglement but also yields new insights into quantum computation.

\section{ACKNOWLEDGMENT}

This research was supported by the National Natural Science Foundation of China under Grant Nos. 60407003 and 61178049.

\section{REFERENCES}

[1] Nielsen MA, Chuang IL. Quantum computation and quantum information. Cambridge: Cambridge University Press; 2000.

[2] Bennett CH, Brassard G, Crépeau C, Jozsa R, Peres A, Wootters WK. Teleporting an unknown quantum state via dual classical and Einstein-Podolsky-Rosen channels. Phys Rev Lett. 1993;70 (13):1895-1899. doi:10.1103/PhysRevLett.70.1895

[3] Ekert A, Jozsa R. Quantum algorithms: entanglement-enhanced information processing. Philos Trans R Soc London. 1998;356: 1769.

[4] Einstein A, Podolsky B, Rosen N. Can quantum-mechanical description of physical reality be considered complete? Phys Rev. 1935;47(10):777-780. doi:10.1103/PhysRev.47.777

[5] Bell JS. On the Einstein-Podolsky-Rosen paradox. Physics. 1964; 1:195.

[6] Tan SM, Walls DF, Collett MJ. Nonlocality of a single photon. Phys Rev Lett. 1991;66(3):252-255. doi:10.1103/PhysRevLett. 66.252

[7] Hardy L. Nonlocality of a single photon revisited. Phys Rev Lett. 1994;73(17):2279-83. doi:10.1103/PhysRevLett.73.2279

[8] Hessmo B, Usachev P, Heydari H, Björk G. Experimental demonstration of single photon nonlocality. Phys Rev Lett. 2004; 92(18):180401. doi:10.1103/PhysRevLett.92.180401
[9] Cerf NJ. Entropic bounds on coding for noisy quantum channels. Phys Rev A. 1998;57(5):3330-47. doi:10.1103/Phys RevA.57.3330

[10] Lee KF, Thomas JE. Experimental simulation of two-particle quantum entanglement using classical fields. Phys Rev Lett. 2002;88:097902.

[11] Lee KF, Thomas JE. Entanglement with classical fields. Phys Rev A 2004;69:052311.

[12] Spreeuw RJC. Classical wave-optics analogy of quantuminformation processing. Phys Rev A. 2001;63(6):062302. doi:10.1103/PhysRevA.63.062302

[13] Bhattacharya N, Van Linden Van Den Heuvell HB, Spreeuw RJC. Implementation of quantum search algorithm using classical Fourier optics. Phys Rev Lett. 2002;88(13):137901. doi:10.1103/ PhysRevLett.88.137901

[14] Goldin MA, Francisco D, Ledesma S. Simulating Bell inequality violations with classical optics encoded qubits. J Opt Soc Am B. 2010;27(4):779. doi:10.1364/JOSAB.27.000779

[15] Francisco D, Ledesma S. Classical optics analogy of quantum teleportation. J Opt Soc Am B. 2008;25(3):383. doi:10.1364/ JOSAB.25.000383

[16] Massar S, Bacon D, Cerf NJ, Cleve R. Classical simulation of quantum entanglement without local hidden variables. Phys Rev A. 2001;63(5):052305. doi:10.1103/PhysRevA.63.052305

[17] Dragoman D. Phase space correspondence between classical optics and quantum mechanics. Prog Opt. 2002;42:424-86.

[18] Lee KF, Reil F, Bali S, Wax A, Thomas JE. Heterodyne measurement of Wigner distributions for classical optical fields. Opt Lett. 1999;24:1370-72.

[19] Jozsa R, Linden N. On the role of entanglement in quantumcomputational speed-up. Proc Roy Soc London A. 2003;459:2011.

[20] Blume-Kohout R, Caves CM, Deutsch IH. Climbing mount scalable: physical resource requirements for a scalable quantum computer. Found Phys. 2002;32(11):1641-70. doi:10.1023/ A:1021471621587

[21] Fu J, Sun S. Efficient simulation of quantum states based on classical fields modulated with pseudorandom phase sequences. http://arxiv.org/abs/1003.6033.

[22] Viterbi AJ. CDMA: principles of spread spectrum communication. (Addison-Wesley Wireless Communications Series). Boston: Addison-Wesley; 1995.

[23] Peterson RL, Ziemer RE, Borth DE. Introduction to spread spectrum communications. Englewood Cliffs (NJ): PrenticeHall; 1995.

[24] Zigangirov K. Theory of CDMA communication. NJ: Wiley IEEE Press; 2004.

[25] Park WJ, Komo JJ. Relationships between m-sequences over GF (q) and $\mathrm{GF}\left(\mathrm{q}^{\mathrm{m}}\right)$. IEEE Trans Info Theory. 1989;35(1):183-186. doi:10.1109/18.42190

[26] Fu J, Si Z, Tang S, Deng J. Classical simulation of quantum entanglement using optical transverse modes in multimode waveguides. Phys Rev A. 2004;70(4):042313. doi:10.1103/ PhysRevA.70.042313

[27] Fu J. Quantum computations with optical waveguide modes. Proc SPIE. 2003;5105:225.

[28] Clauser JF, Horne MA, Shimony A, Holt RA. Proposed experiment to test local hidden-variable theories. Phys Rev Lett 1969;23:880.

[29] Greenberger DM, Horne MA, Shimony A, Zeilinger A. Bell's theorem without inequalities. Am J Phys. 1990;58(12):1131. doi:10.1119/1.16243 


\section{COMPETING INTERESTS}

The authors declare no competing interests.

\section{PUBLISHING NOTES}

(C) 2015 J. Fu and X. Wu. This work has been published open access under Creative Commons Attribution License CC BY 4.0, which permits unrestricted use, distribution, and reproduction in any medium, provided the original work is properly cited. Conditions, terms of use and publishing policy can be found at www.scienceopen.com.
Please note that this article may not have been peer reviewed yet and is under continuous post-publication peer review. For the current reviewing status please click here or scan the QR code on the right.

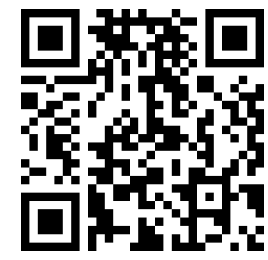

